\title{
THE ANALYSIS OF METHYLATION OF DNA PROMOTER OF SFRP2 GENE IN PATIENTS WITH HYPERPLASTIC PROCESSES OF THE ENDOMETRIUM
}

\author{
V.G. Marichereda ${ }^{1}$, N.A. Bykova ${ }^{1}$, V.V. Bubnov', G.S. Manasova ${ }^{1}$, T.Y. Moskalenko², A.G. Volyanska ${ }^{1}$, \\ I.M. Shevchenko', T.M. Adamovska ${ }^{1}$ \\ ${ }^{1}$ Odesa National Medical University, Odesa 65082, Ukraine \\ ${ }^{2}$ Maternity Hospital № 7, Odesa 65082, Ukraine
}

\begin{abstract}
The hyperplastic processes of the endometrium can arise not only against the background of excessive influence of estrogen, but also against the background of epigenetic damages that affect apoptosis, cell proliferation, differentiation, and adhesion, and DNA reparation. The aim of our study was to investigate and analyze the status of methylation of the promoter of SFRP2 gene in patients with hyperplastic processes of the endometrium. Materials and Methods: The study groups were the following: I - patients with endometrial hyperplasia $(\mathrm{EH}, \mathrm{n}=9)$; II - patients with endometrial intraepithelial neoplasia $(\mathrm{EIN}, \mathrm{n}=10)$, III - control groups: 1) with endometrial cancer $(\mathrm{EC}, \mathrm{n}=4)$, and 2$)$ healthy women $(\mathrm{n}=4)$. Determination of promoter methylation of $S F R P 2$ gene was carried out by the semiquantitative method of methylation-specific PCR assay. Results: The maximum level of methylation of SFRP2 gene promoter had been revealed in patients with $\mathrm{EC}-\mathbf{4 2 . 8 0} \pm 3.55 \%(p<0.05)$. The patients of the I group had the lowest values of methylation of SFRP2 gene promoter $-10.66 \pm 0.85 \%$, while in patients of the II group this indicator was higher $-20.60 \pm 0.95 \%(p<\mathbf{0 . 0 5})$. In healthy women of the control group, methylation of $S F R P 2$ gene promoter was detected in none of the samples. Conclusion: The content of the methylated SFRP2 gene in endometrial tissue of patients with hyperplastic processes higher than 20-25\% allows relate these women to the risk group of EC development and dictates the need of intensive observation of such patients.
\end{abstract}

Key Words: methylation, SFRP2 gene, hyperplastic processes of the endometrium.

Hyperplastic processes of the endometrium (HPE) are related to the most actual problems in gynecology and require attention of obstetricians-gynecologists due to high risk of a malignization and recurrence [1, 2]. HPE is considered to be the pathology of the endometrium, which is characterized by diffuse or focal enlargement of the endometrium, and can arise not only at the background of excessive exposure to estrogen, but also via epigenetic disorders that affect apoptosis, inactivation of cell proliferation, differentiation, cell adhesion, DNA repair and as a result, lead to an imbalance of proliferation and apoptosis [3-6].

Also the tendency of "rejuvenation" of this pathology is supervised for last years. It is known, that endometrial cancer (EC) develops against the background of an endometrial hyperplasia (EH) in about 50\% of cases. Different morphological types of the HPE (from a simple $\mathrm{EH}$ without atypia to an $\mathrm{EC}$ ) represent the progressing changes in histo- and cytoarchitectonics with accumulation of the nosospecific molecular and genetic changes of cells $[7,8]$.

An abnormal methylation of DNA is the earliest event, which occurs in cell before the morphological manifestation of an $\mathrm{EH}$. It is revealed that methylation of CpG-islands is the cause of loss of an expression of the corresponding genes due to the blocking of their

\section{Submitted: January 16, 2017.}

*Correspondence: E-mail: dimitrovanatalie@gmail.com Abbreviations used: EC - endometrial cancer; $\mathrm{EH}$ - endometrial hyperplasia; EIN - endometrial intraepithelial neoplasia; HPE hyperplastic processes of the endometrium; PE - proliferative endometrium; SE - secretory endometrium. transcription [7, 9]. Determination of the status of DNA methylation of nosospecific genes helps with early and differential diagnosis of tumors, and also is an important event for observation and the prognosis.

The study [10] showed that methylation of FOXP3 gene promoter in patients with $\mathrm{EC}$ is around $56-77 \%$. Another study [11] showed that the development of HPE is not associated with WIF1 gene methylation.

SFRPs family secreting proteins are antagonists of a Wnt-regulatory pathway, they suppress this signaling pathway in healthy individuals. The Wnt-signaling pathway promotes the regulation of proliferation and differentiation of cells. It is proved that in some malignant tumors, such as a colorectal cancer, tumors of head and neck, gastric cancer, methylation of genes-antagonists of a Wnt-signaling pathway is the reason of uncontrollable cellular proliferation [12, 13].

Traditionally, ultrasound, endoscopic and pathomorphological methods of a research are used for diagnostics of HPE [14]. But none of these methods shows an exact assessment; even the discrepancy an atypical hyperplasia and EC in differential diagnostics by morphologists constitutes $11-20 \%$ of cases [7]. There is a need for search of more reliable, early criteria of HPE diagnostics by determination of a condition of DNA methylation. Therefore, research of the methylation status of SFRP2 gene in patients with HPE and patients with $\mathrm{EC}$ is actual for modern gynecologic practice.

The aim of our study was to investigate and analyze the DNA methylation status of the promoter of SFRP2 gene in patients with HPE. 


\section{MATERIALS AND METHODS}

$27(100 \%)$ patients at the age of $18-76$ years, have participated in a research, criteria of inclusion were the following: the informed patient's consent to participation in a research; age - reproductive, peri- and postmenopausal; pathomorphologically confirmed diagnosis of HPE. Criteria of an exclusion were the age $<18$ years or pregnancy.

Involved in the research patients were treated in the Gynecology Department of Multidisciplinary Medical Center University Clinic \# 1 of Odesa National Medical University in a planned or urgent order and were operated thereon due to abnormal uterine bleeding, HPE. Diagnoses were confirmed by ultrasound diagnostics before an operative intervention. Preoperative examination and preparation of patients were carried out by standard principles according to the order of Ministry of Health Care of Ukraine No. 620 from 29.12.2003. The extent of an operative intervention was chosen individually for each patient. It depended on a clinical situation: a hysteroscopy with a biopsy of endometrium, curettage of a mucous cavity of the uterus, a hysterectomy with/without uterus appendages, pipelle biopsy of the endometrium. The existence of HPE was confirmed pathomorphologically in all patients who participated in a research according to EINclassification of HPE: EH, endometrial intraepithelial neoplasia (EIN) and classification of histopathological subtypes of EC: endometrioid adenocarcinoma, mucinous carcinoma, serous carcinoma, clear cell carcinoma, squamous cell carcinoma, undifferentiated carcinoma, mixed carcinoma, metastatic carcinoma [1, 15-17].

Depending on the HPE morphological type, examined patients were distributed into the groups: I) EH 9 (33.3\%) patients; II) EIN - 10 (37.1\%) patients; III) control groups: 1) EC - 4 (14.8\%) patients, and 2) healthy women $-4(14.8 \%)$.

The age of patients of the I group was 18-59 years, among them $6(66.7 \%)$ patients were of reproductive age, $2(22.2 \%)$ patients - of perimenopausal age, $1(11.1 \%)$ patient of postmenopausal age. $3(33.3 \%)$ patients, mainly perimenopausal and postmenopausal age, were observed by the therapist because of an idiopathic hypertension. $2(22.2 \%)$ patients had high index of body weight - 29.2; 33.4. A hysteroscopy with a "cold" curettage of endometrium was carried out to $5(55.6 \%)$ patients, indications to which were sterility in $2(40.0 \%)$ cases and HPE according to an ultrasound examination in $3(60.0 \%)$ cases. The hysteroscopy was carried out with the use of the endoscopic equipment Karl Storz (Germany). The fractional diagnostic curettage of endometrium was carried out to $3(33.3 \%)$ women, due to an abnormal uterine bleeding. 1 (11.1\%) patient underwent a hysterectomy without appendages (basic disease is hysteromyoma with a hemorrhagic syndrome). USE of organs of the small pelvis, which was carried out by the device ALOKA Pro Sound SSD 5000 (Japan), showed disharmony of thickness of endometrium to a phase of a menstrual cycle, heteroge- neity and hyperechogenicity of the endometrium. USE of organs of a small pelvis had shown the existence of non-uniform hyperechoic-thickened endometrium (11-19 $\mathrm{mm}$ - in reproductive age, 5-11 $\mathrm{mm}$ - in postmenopausal period). Also it was established that $1(11.1 \%)$ patients had polycystic ovaries, 3 (33.3\%) hysteromyoma, 1 (11.1\%) - adenomyosis.

The age of patients of the II group was 39-64 years: $2(20.0 \%)$ patients of reproductive age and 4 (40.0\%) patients of perimenopausal and postmenopausal age. $5(50.0 \%)$ women had high index of body weight 28.3-37.4. 5 (50.0\%) patients had normal weight. A half of patients were observed by the therapist because of a hypertension. 7 (70.0\%) patients had appealed because of menstrual disorders, which turned into abnormal uterine bleeding. $3(30.0 \%)$ patients with a disease of endometrium had no symptoms. USE of organs of a small pelvis had shown the existence of non-uniform hyperechoic thickened endometrium (14-23 $\mathrm{mm}$ in reproductive age, 9-17 $\mathrm{mm}$ - in postmenopausal period). Also ultrasound revealed uterine myoma in 5 (50.0\%) women and 1 (10.0\%) patient - adenomyosis. 4 (40.0\%) patients had an operative intervention - a hysterectomy with appendages, 2 (20.0\%) women had a hysteroscopic resection of the endometrium, and 4 (40.0\%) patients the dilation and curettage of the uterus.

The age of patients of the control group with EC was 49-72 years. $3(75.0 \%)$ patients were at postmenopause, $1(25.0 \%)$ - of reproductive age. The index of body weight of patients with EC was more than 30 , that confirmed an obesity; 2 (50.0\%) patients were on the regular medical check-up at the therapist because of an idiopathic hypertension, 1 (25.0\%) - under observation of the endocrinologist because of a ll type diabetes mellitus. All patients with EC had complained on menstrual disorders in the form of a heavy irregular menses and abnormal uterine bleedings. 2 (50.0\%) patients underwent the uterus curettage, 2 (50.0\%) patients - a hysteroscopy for biopsy of endometrium. According to results of USE of organs of the small pelvis, which were carried out before an operative intervention, all 4 (100\%) patients had the enlargement of the endometrium (in reproductive age - 18-28 mm, 11-24 mm - at postmenopausal period), its heterogeneity, in equality of an external contour, absence of accurate margins between the tumor center and a myometrium. The hysteromyoma had been diagnosed in 2 (50.0\%) patients, and adenomyosis had been diagnosed in 1 (25.0\%) patient.

The healthy women of the control group (4 (100.0\%)) without pathology of the endometrium participated in the study and were examined in the clinic for preparation to the in vitro fertilization program because of male infertility factor. The age of the examined control group was 29-36 years. The absence of the pathology of the endometrium is confirmed morphologically (pipelle biopsy of the endometrium) and by ultrasound of the pelvic organs (M-echo 5-13 $\mathrm{mm}$ ). Genital and extragenital pathology in the control group women were not detected. 
Determination of methylation of the DNA promoter of SFRP2 gene was carried out by the semiquantitative method of methylation-specific PCR assay.

DNA from samples of endometrium tissues were isolated with use of DNeasy Blood \& Tissue Kit (Qiagen, USA). Bisulfite processing of DNA was carried out with using EpiTect Bisulfite Kit (Qiagen, USA) according to the protocol of the manufacturer. The amount of DNA for bisulfite processing of DNA in all tests was $1 \mu \mathrm{g} / \mathrm{ml}$. Amplification of DNA after bisulfite processing was carried out using Hot Start DNA Polymerase Kit according to the program $95^{\circ} \mathrm{C}-15 \mathrm{~min} ; 95^{\circ} \mathrm{C}-30 \mathrm{~s}, 50^{\circ} \mathrm{C}-$ for a unmethylated DNA and $56^{\circ} \mathrm{C}-$ for the methylated DNA, $72^{\circ} \mathrm{C}-30 \mathrm{~s}, 39$ cycles; $72^{\circ} \mathrm{C}-10 \mathrm{~min}$ (Table 1 ).

Table 1. The sequence of primers for amplification of the methylation-specific site of SFRP2 promoter

\begin{tabular}{llc}
\hline \multicolumn{1}{c}{ Type } & \multicolumn{1}{c}{ Sequence of primers } & $\mathrm{t},{ }^{\circ} \mathrm{C}$ \\
\hline For methylated & F_M_GGGTCGGAGTTTTTCGGAGTTGCGC & 56 \\
SFRP2 gene & R_M_CCGCTCTCTTCGCTAAATACGACTCG & \\
For unmethylated & F_U_TTTGGGTTGGAGTTTTTTGGAGTTGTGT & 50 \\
SFRP2 gene & R U AACCCACTCTCTTCACTAAATACAACTCA & \\
\hline
\end{tabular}

The PCR products were examined using $2 \%$ agarose gel electrophoresis, stained with ethidium bromide. The relative content the methylated and unmethylated DNA was assessed using the Quantity One 1D Analysis Software program (BIO-RAD, USA) on the device Versa Doc MP 4000 System (BIO-RAD, USA) according to the formula:

$$
M(\%)=A \cdot 100 /(A+B) \text {, }
$$

where $\mathrm{A}$ - is the area of peak of methylated DNA, B is the area of peak of unmethylated DNA, $\mathrm{M}$ - relative content the methylated DNA.

The molecular-biological studies of the DNA methylation were controled by EpiTech Control DNA unmethylated (Qiagen, USA) and EpiTect Control DNA methylated (Qiagen, USA).

Statistical analysis of the data obtained was validated using MedCalc 14.8.1 and Microsoft Excel
2010 packages with the AtteStat add-in 12.5. The average sample values of the quantitative attributes are given as $M \pm m$, where $M$ is the mean selective, $m$ is the mean error. Taking into account the small number of observations in the groups for comparison of the main parameters, nonparametric methods were used - the medication test of Kruskal - Wallis, the Mann - Whitney test and the Wald - Wolfowitz series.

\section{RESULTS AND DISCUSSION}

The analysis of the obtained data on methylation of the promoter of SFRP2 gene has revealed significant differences between the patients with EH, EIN, EC and healthy women (Table 2, Fig. 1, 2).

Table 2. The content of methylated DNA of a gene SFRP2 in the studied samples of endometrium

\begin{tabular}{ccc}
\hline Case & $\begin{array}{c}\text { Morphological type of the } \\
\text { endometrium }\end{array}$ & $\begin{array}{c}\text { The content of SFRP2 gene } \\
\text { methylation, } \%\end{array}$ \\
\hline 1 & EH & 8 \\
2 & EH & 12 \\
3 & EH & 16 \\
4 & EH & 9 \\
5 & EH & 11 \\
6 & EH & 11 \\
7 & EH & 12 \\
8 & EH & 8 \\
9 & EH & 9 \\
10 & EIN & 22 \\
11 & EIN & 17 \\
12 & EIN & 17 \\
13 & EIN & 19 \\
14 & EIN & 24 \\
15 & EIN & 26 \\
16 & EIN & 23 \\
17 & EIN & 19 \\
18 & EIN & 20 \\
19 & EIN & 19 \\
20 & EC & 33 \\
21 & EC & 45 \\
22 & EC & 49 \\
23 & EC & 51 \\
24 & PE & 0 \\
25 & PE & 0 \\
26 & PE & 0 \\
27 & SE & 0 \\
\hline
\end{tabular}

Note: PE - proliferative endometrium; SE - secretory endometrium.

The level of DNA methylation of SFRP2 gene (\%)

$$
60
$$

50

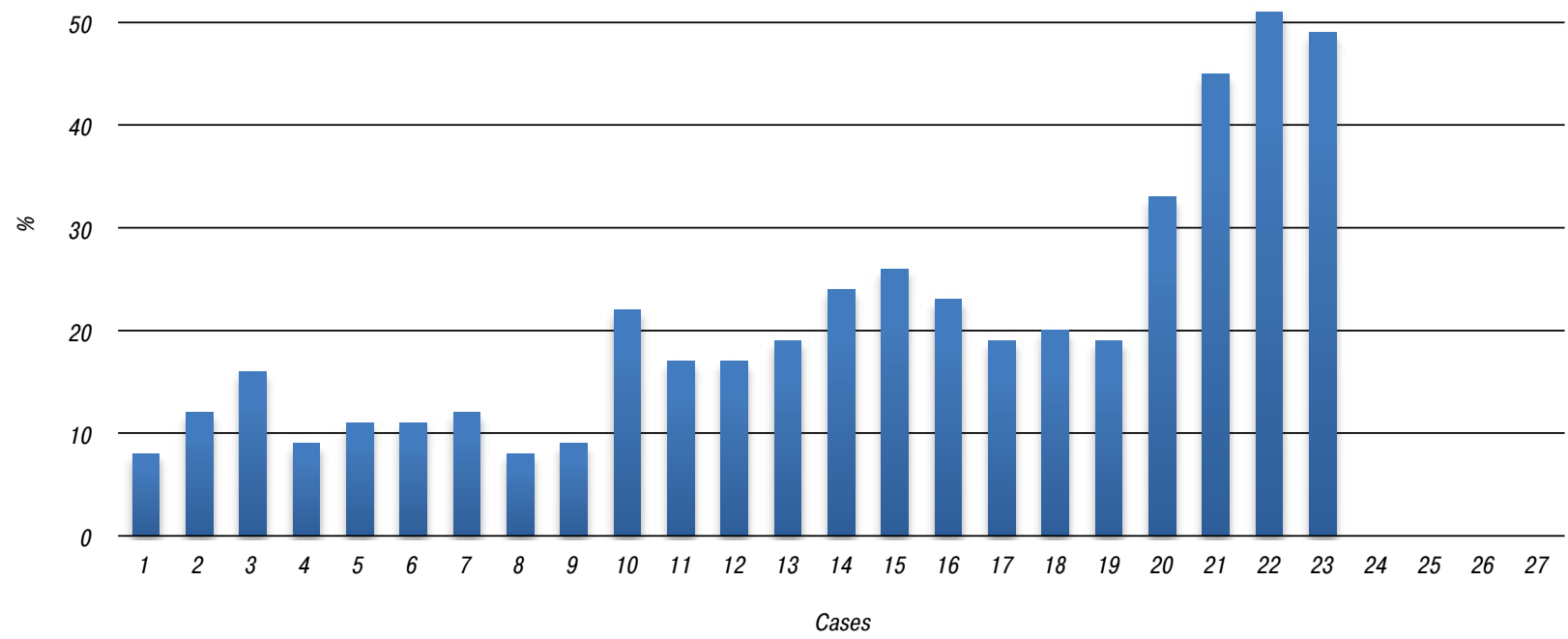

Fig. 1. The level of methylated SFRP2 gene in the studied endometrial samples. Cases 1-9 - patients with EH, 10-19 - patients with EIN , 20-23 - patients with EC, 24-27 - healthy women 
The maximum level of methylation of the studied gene had been found in patients with EC $42.80 \pm 3.55 \%(p<0.05)$, that is nearly by 4 -fold higher, than in patients with $\mathrm{EH}$ and by 2 -fold than in patients with EIN. It was revealed that patients with EH had the lowest values of this index; the average content of the methylated SFRP2 gene was $10.66 \pm 0.85 \%$, while in patients with EIN this index was $20.60 \pm 0.95 \%(p<0.05)$ (Fig. 1, 2, Table 2). In healthy women of the control group, methylation of the investigated gene was detected in none of the cases, therefore, the graphic representation of the results was not performed (Table 2).

It is necessary to note that methylation of the promoter of SFRP2 gene didn't depend on the age of the patients, existence of the accompanying gynecologic pathology (hysteromyoma, adenomyosis, polycystic ovaries).

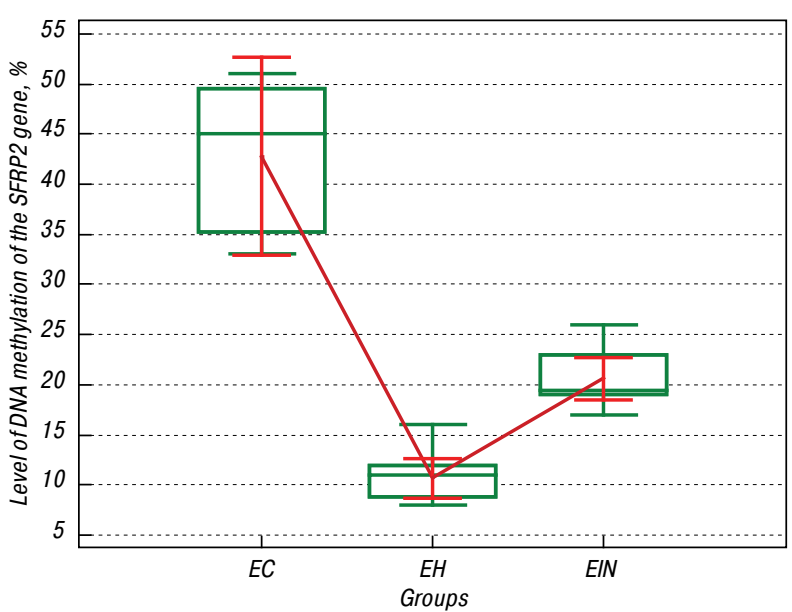

Fig. 2. Methylation of the promoter of SFRP2 gene in patients with $\mathrm{EH}, \mathrm{EIN}$ and EC

Also, an assessment of the intensity of methylation of the promoter of SFRP2 gene confirmed the results (Fig. 3) shown above. The methylation of DNA in control sample (№ 1) was taken as $50 \%$. In comparison with the control, the minimum intensity of methylation was noted in patients with $\mathrm{EH}$ (samples № 3, № 4, № 5). The maximal intensity of DNA methylation was found in patients with EC (samples № 6, № 7), being higher in comparison with patients with $\mathrm{EH}$ (№ 3, № 4, № 5) and the EIN (№ 2).

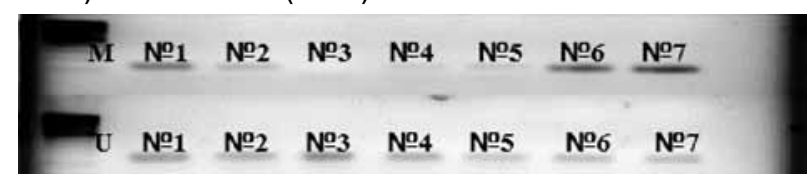

Fig. 3. Intensity of methylation of the promoter of SFRP2 gene in endometrial samples from different groups under study. $M-$ methylated DNA; U — unmethylated DNA

The dependence between the level of DNA methylation and a morphological stage of HPE has shown its lowest level in patients with $\mathrm{EH}$, and the highest in patients with EC. Average values of DNA methylation were characteristic for patients with EIN.

The data on the status of SFRP2 methylation in patients with HPE and EC in the literature are scarce. In the paper of Di Domenico et al. [18] in has been shown that methylation of SFRP2 gene could not serve as a diagnostic criterion for the diagnosis of EC, as it was detected in 2 out of 39 examined patients with HPE and EC. There is a high incidence of methylation of another gene from the Secreted Frizzled Related Protein family, SFRP1, among the EC specimens and this index allow clearly distinguish benign and malignant areas of the mucosal tissue of the uterine cavity. The results of our study showed a high frequency of SFRP2 gene methylation among patients with HPE, with a direct relationship between the degree of methylation and the morphological type of the endometrium pathology: $\mathrm{EH} \rightarrow \mathrm{EIN} \rightarrow \mathrm{EC}$. There are some data that the state of SFRP2 gene methylation could also be used to diagnosis of oncological processes of other localizations (colorectal, ovarian, lung cancer), not only EC [19-21].

\section{CONCLUSION}

Thus, the presence of not only morphological changes in case of HPE, but also characteristic changes of SFRP2 methylation in different types of HPE, expands diagnostic criteria of this nosology. Low content of the methylated DNA (10.66 $\pm 0.85 \%)$ in case of $\mathrm{EH}$ and high content of the methylated DNA $(42.80 \pm 3.55 \%)$ in patients with $E C$, allow to use determination of relative content of the methylated SFRP2 gene as a marker of differential diagnostics of HPE and early prognosis of risk of its malignization. The SFRP2 gene can be used as a marker for the differential diagnosis of HPE and EC. The content of the methylated SFRP2 gene in endometrial tissue of patients with hyperplastic processes higher than $20-25 \%$ allows relate these women to the risk group of EC development and dictates the need of intensive observation of such patients.

\section{REFERENCES}

1. Tatarchuk TF, Kalugina LV, Tutchenko TM. Hyperplastic processes of endometrium: what's new? Repr Endocrinol 2015; 5: 7-13 (in Russian).

2. Sidorova I, Unanyan A, Vlasov R, et al. Endometrial hyperplastic processes: clinical and therapeutic features. Doctor 2011; 6: 58-60 (in Russian).

3. Gulyaeva LF, Krasilnikov SE. Molecular mechanisms of endometrial carcinoma. Bull ESSC SD RAMS 2012; 3: 110-5 (in Russian).

4. Delpu Ya, Cordelier P, C. Cho W. DNA methylation and cancer diagnosis. Int J Mol Sci 2013; 14: 15029-58.

5. Gros C, Fahy J, Halby L, et al. DNA methylation inhibitors in cancer: Recent and future approaches. Biochimie 2012; 94: 2280-96.

6. Sivova EN, Pachov AI, Bukreev AV, Fokina AP. Some questions of etiology and pathogenesis of endometrial proliferative processese. Siber Med Rev 2015; 1: 16-22 (in Russian).

7. Stanoyevich IV, Zemlyakova VV, Fen I, et al. Abnormal methylation of a number of genes in endometrial hyperplasia in the presence of chronic endometritis. Russ Bull Obst Gynecol 2012; 1: 20-3 (in Russian).

8. Matias-Guiu X, Catasus L, Bussaglia E, et al. Molecular pathology of endometrial hyperplasia and carcinoma. Hum Pathol 2001; 32: 569-77. 
9. Prat J, Gallardo A, Cuatrecasas M, et al. Endometrial carcinoma: pathology and genetics. Pathology 2007; 39: 72-87.

10. Buchynska LG, Iurchenko NP, Verko NP, et al. FOXP3 gene promoter methylation in endometrial cancer cells. Exp Oncol 2015; 4: 246-9.

11. Zaporozhan VN, Dubinina VG, Bubnov VV, et al. Analysis of gene WIF1 methylation in women with hyperproliferative processes of endometrium and endometrial carcinoma. Ach Biol Med 2015; 2: 55-7 (in Russian).

12. Ma XY, Ma CX, Wang JN. Endometrial carcinogenesis and molecular signaling pathways. Am J Mol Biol 2014; 4: $134-49$.

13. Komiya $Y$, Habas R. Wnt signal transduction pathways. Organogenesis 2008; 4: 68-75.

14. Zaporozhan VN, Tatarchuk TF, Dubinina VG, et al. Modern diagnostics and treatment of endometrial hyperplastic processes. Repr Endocrinol 2012; 1: 5-12 (in Russian).

15. Khanna R, Rupala $G$, Khanna V, et al. Endometrial intraepithelial neoplasia and its correlation with WHO classified endometrial hyperplasia. Int J Pathol 2010; 1: 1-4.
16. Ordi J, Bergeron C, Hardisson D, et al. Reproducibility of current classifications of endometrial endometrioid glandular proliferations: further evidence supporting a simplified classification. Histopathology 2013; 64: 284-92.

17. Salman MC, Usubutun A, Boynukalin K, Yuce K. Comparison of WHO and endometrial intraepithelial neoplasia classifications in predicting the presence of coexistent malignancy in endometrial hyperplasia. J Gynecol Oncol 2010; 21: 97-101.

18. Di Domenico M, Santoro A, Ricciardi C, et al. Epigenetic fingerprint in endometrial carcinogenesis: the hypothesis of a uterine field cancerization. Cancer Biol Ther 2011; 5: 447-57.

19. Shunlin L, Xiaoying C, Ruhua C, et al. Diagnostic role of Wnt pathway gene promoter methylation in non small cell lung cancer. Oncotarget 2017; 22: 36354-67.

20. Ashktorab H, Brim H. DNA methylation and colorectal cancer. Curr Colorectal Cancer Rep 2014; 10: 425-30.

21. Nikbakht M, Shabanizadeh A, Salehi M, et al. BRCA1 promoter methylation status in ovarian cancer. Lab Med 2012; 43: 18-21. 